\title{
Symptomatic treatment of uncomplicated lower urinary tract infections in the ambulatory setting: randomised, double blind trial
}

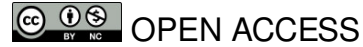

An error occurred in the key for figure 2 of this Research paper by Kronenberg and colleagues (BMJ 2017;359:j4784, doi:10.

1136/bmj.j4784). The blue line represents norfloxacin [not diclofenac] and the broken line represents diclofenac [not norfloxacin]. 